\title{
Towards an optimization of the formulation of geopolymers in the fresh state: Rheological approach
}

https://doi.org/10.1515/arh-2019-0009

Received May 15, 2019; accepted Sep 02, 2019

\begin{abstract}
In this work, we are interested in describing the evolution of the rheological behavior of geopolymer materials since their elaboration until the start of their kinetics of setting. We considered the case where these materials are obtained from the dissolution of a quantity $\mathrm{S}$ of metakaolin in a quantity $\mathrm{L}$ of alkaline activation solution (sodium-based). In this context and for all the values of the structural identification parameters $\mathrm{P}=\left(\% \mathrm{Na}_{2} \mathrm{O}\right)$ and $\mathrm{R}_{3}=(\mathrm{L} / \mathrm{S})$ in our experiments, it has been established that the rheological behavior is described by the viscoplastic Herschel-Bulkley model. In particular, we have been able to determine how rheological parameters of the model are affected by variations of $\mathrm{P}$ and / or $\mathrm{R}_{3}$. For the considered formulations, this analysis is of importance insofar as it was at the base of the identification of critical values of $P$ and R3. Moreover, we verified that for these values, the setting time is maximum. This result is retained to define the optimized formulation of our materials.
\end{abstract}

Keywords: Rheological behavior, Geopolymers, Optimized formulation, Setting time, Herschel-Bulkley

PACS: 47.57.Qk

\section{Introduction}

For several years, the characterization of geopolymers has been an area of investigation that is attracting more and

\footnotetext{
^Corresponding Author: Nouha Lahlou: Department of Physics, Faculty of Sciences Ain Chock, Hassan II University, 20470 Casablanca, Morocco; Email: nouhalahlou10@gmail.com Mohamed Ouazzani Touhami: Department of Physics, Faculty of Sciences Ain Chock, Hassan II University, 20470 Casablanca, Morocco
}

Rabii Hattaf, Redouane Moussa: Department of Chemistry, Faculty of Sciences Ain Chock, Hassan II University, 20470 Casablanca, Morocco

O̊pen Access. (c) 2019 N. Lahlou et al., published by De Gruyter. (Cc) BY License more interest both academically and industrially. These interests are justified by the multiple performances that these materials exhibit, in particular their fluidity in the fresh state, sufficient for their pumping and injection, their resistance to attacks by acidic media, the possibility of adapting their setting time or still the importance of their mechanical strength in the hardened state [1].

For these materials, obtained from the activation of a natural or synthetic aluminosilicate powder by an alkaline solution, several techniques have been implemented for the apprehension of their microscopic and / or macroscopic properties. We can cite in this context the characterization techniques which are based on various basic physical principles including in particular the interactions between matter and radiation (X-ray diffraction, infrared, X-ray fluorescence), thermal transfer (differential thermal analysis, thermo-gravimetric analysis, differential scanning calorimetry analysis) or the various mechanical tests (compression, flexion, traction).

The exploitation of these different techniques has been the basis of several studies to highlight the impact of the structural parameters of formulation on the texturing of geopolymers, their structuring or their resistance to compression [2, 3]. For almost all of this work, it should be noted that the only parameter used to identify a given formulation is the value of the silicium / aluminum ratio ( $\mathrm{Si}$ / $\mathrm{Al})$. It should also be noted that the start of the transition from the fresh state to the hardened state has been defined through the measurement of a characteristic time (setting time) taken with the aid of the needle VICAT [4]. Results obtained made it possible to study the evolution of setting time as a function of the structural composition.

The importance of these results and the need for greater control over the processes used to utilize the materials under consideration have been the basis of several additional works [5-9]. For these studies, deployed for twenty years, a more detailed description of the evolution of these materials according to their formulation is proposed. It is based on the introduction of the structural identification parameters $\mathrm{R}_{1}=\left(\% \mathrm{SiO}_{2}\right) /\left(\% \mathrm{Na}_{2} \mathrm{O}\right), \mathrm{R}_{2}$ 
$=\left(\% \mathrm{H}_{2} \mathrm{O}\right) /\left(\% \mathrm{Na}_{2} \mathrm{O}\right)$ and $\mathrm{R}_{3}=\mathrm{L} / \mathrm{S}(\mathrm{L}=$ Quantity of the solution of alkaline activation and $\mathrm{S}=$ amount of the aluminosilicate powder). It also includes considering results deduced from experimental rheology in characterizing the evolution [5] of these solutions. It has been established in particular that the rheological models of the yield fluids make it possible to correctly describe the behavior of the geopolymer materials in the fresh state and that the transition to the hardened state can be noted from the temporal changes of viscosity.

In spite these advances, no one has dwelt on how changes in parameters $R_{1}, R_{2}$ and $R_{3}$ affect rheological variables such as yield stress, flow index and consistency, and therefore the start of the kinetics of setting.

The precision of this double correspondence between parameters of a structural nature and parameters with a rheological connotation on the one hand and between these rheological parameters and the phenomenon of setting, on the other hand, takes our particular significance. This insofar as it becomes more and more opportune to introduce, at the level of behavioral laws, empirical models capable alone to facilitate the identification of the various correlations sought and consequently to lead to important industrial applications $[9,10]$.

Our work is part of this framework and focused, initially, on the description of this correspondence that enriches the different means of control and prediction described above. Indeed, the apprehension of the kinetics of setting is dependent mainly on the rheological parameters. In a second step, we paid particular attention to the determination of an optimized formulation of geopolymer materials in the fresh state. The objective is to deduce, from a purely rheological approach, the composition for which the setting time is extreme.

The results obtained were the basis for the introduction of a new approach for the control of geopolymer materials, based entirely on the evolution of their rheology in the fresh state. In fact, it is a question of specifying the optimized formulation of these matrices for a setting time previously stopped. Thus, this enriches the reflection around this problem often addressed from the only data of the mechanical strength of the final product.

\section{Formulation and protocol for elaboration of geopolymer matrices based on Metakaolin}

\subsection{Description of the raw materials}

For the tests conducted as part of this work, we consider the following products:

- The Sodium hydroxide pellets of $98.5 \%$ purity (Cadilhac ${ }^{1}$ company)

- An alkaline solution of sodium silicate with $45 \%$ by weight of dry matter, an $\mathrm{SiO}_{2} / \mathrm{Na}_{2} \mathrm{O}$ molar ratio of 2 $\pm 0.1\left(15 \% \mathrm{Na}_{2} \mathrm{O}, 30 \% \mathrm{SiO}_{2}\right.$ and $55 \% \mathrm{H}_{2} \mathrm{O}$ ). (company Cadilhac1)

- The artificial pozzolan (Metakaolin) "ARGICAL-M 1000 ", obtained by calcination and grinding of a kaolinic clay of the Charentes basin. It is an amorphous material, not crystallized, whose particles have a lamellar form. Its chemical composition is described by the manufacturer: $\mathrm{SiO}_{2}: 55 \% ; \mathrm{Fe}_{2} \mathrm{O}_{3}$ : 1.4\%; $\mathrm{Al}_{2} \mathrm{O} 3: 40 \% ; \mathrm{TiO}_{2}: 1.5 \% ; \mathrm{K}_{2} \mathrm{O}+\mathrm{Na}_{2} \mathrm{O}: 0.8 \%$; $\mathrm{CaO}+\mathrm{MgO}: 0.3 \%$. It is a dehydroxylated alumina silicate of general composition: $\mathrm{Al}_{2} \mathrm{O}_{3} 2 \mathrm{SiO}_{2}$. The X-ray diffraction analyzes on this metakaolin have shown that it is a largely amorphous powder containing a small amount of quartz as impurity phase, and a small residual amount of kaolin (Figure 1) on the test and on the diffractometer.

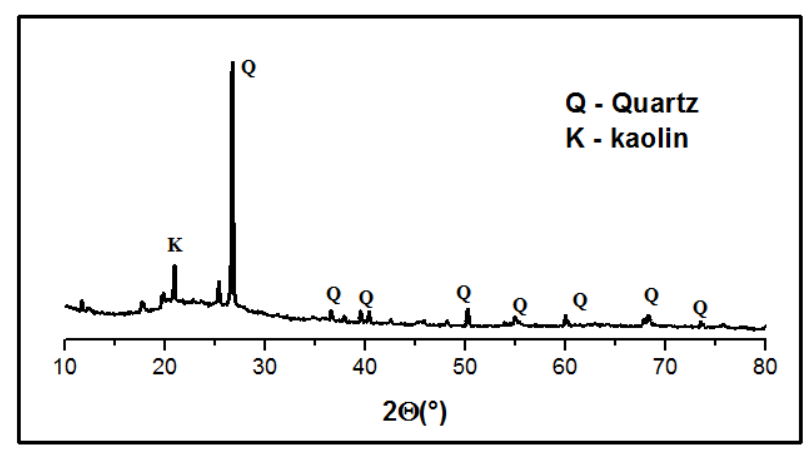

Figure 1: XRD of Metakaolin. 
Table 1: Formulation of activation solution.

\begin{tabular}{|c|c|c|c|c|c|c|c|c|c|c|}
\hline $\mathrm{Q}(\mathrm{Na} 2 \mathrm{O})(\mathrm{g})$ & $\mathrm{Q}(\mathrm{NaOH})$ & $\mathrm{Q}(\mathrm{H} 2 \mathrm{O})$ & $\mathrm{Q}(\mathrm{Sc})$ & $\% \mathrm{SiO} 2$ & $\% \mathrm{Na2O}$ & $\% \mathrm{H} 2 \mathrm{O}$ & Somme $\%$ & Q1 (g) & $\mathrm{R} 2=\mathrm{H} 2 \mathrm{O} / \mathrm{Na} 2 \mathrm{O}$ & $\mathrm{R} 1=\mathrm{SiO} 2 / \mathrm{Na} 2 \mathrm{O}$ \\
\hline 25 & 32,2684737 & 73 & 100 & 15,1515152 & 20,2020202 & 64,6464646 & 100 & 198 & 3,2 & 0,75 \\
\hline 19,88372093 & 25,664693 & 63,6046512 & 100 & 16,3498099 & 19,0114068 & 64,6387833 & 100 & 183,488372 & 3,4 & 0,86 \\
\hline 15 & 19,3610842 & 54,5 & 100 & 17,699115 & 17,699115 & 64,6017699 & 100 & 169,5 & 3,65 & 1 \\
\hline 12,27272727 & 15,8408871 & 49,4545455 & 100 & 18,549747 & 16,8634064 & 64,5868465 & 100 & 161,727273 & 3,83 & 1,1 \\
\hline 10 & 12,9073895 & 45,425 & 100 & 19,3019141 & 16,0849284 & 64,6131575 & 100 & 155,425 & 4,017 & 1,2 \\
\hline 6,428571429 & 8,29760752 & 38,6428571 & 100 & 20,6794682 & 14,7710487 & 64,549483 & 100 & 145,071429 & 4,37 & 1,4 \\
\hline 5 & 6,45369474 & 36 & 100 & 21,2765957 & 14,1843972 & 64,5390071 & 100 & 141 & 4,55 & 1,5 \\
\hline 2,142857143 & 2,76586917 & 30,7142857 & 100 & 22,5806452 & 12,9032258 & 64,516129 & 100 & 132,857143 & 5 & 1,75 \\
\hline 0 & 0 & 26,75 & 100 & 23,6686391 & 11,8343195 & 64,4970414 & 100 & 126,75 & 5,45 & 2 \\
\hline
\end{tabular}

\section{Formulation and development protocols}

\subsection{Case of alkaline activation solutions}

\subsubsection{Formulation}

Our formulation is based on the definition of relations, allowing the passage of the weightings of the quantities considered to molar ratios $R_{1}$ and $R_{2}$. It aims to specify these quantities for the different cases encountered in the previous work or only the ratios $\mathrm{R}_{1}$ and $\mathrm{R}_{2}$ are introduced [11, 12]. It also makes it possible to solve the problems related to calculating these ratios when the activation solution is prepared from a commercial solution of sodium silicate containing $\left(\mathrm{SiO}_{2}, \mathrm{Na}_{2} \mathrm{O}, \mathrm{H}_{2} \mathrm{O}\right)$ with a predefined $\mathrm{SiO}_{2} / \mathrm{Na}_{2}$ $\mathrm{O}$ ratio. In this context and taking into account their definition, the data of $R_{1}$ and $R_{2}$ allowsto specify the percentage $\mathrm{P}$ in $\mathrm{Na}_{2} \mathrm{O}$ through:

$$
\mathrm{P}=\left(\% \mathrm{Na}_{2} \mathrm{O}\right)=\frac{100}{\mathrm{R} 1+\mathrm{R} 2+1}
$$

and to deduce the levels of $\mathrm{SiO}_{2}$ and $\mathrm{H}_{2} \mathrm{O}$ in the alkaline activation solution. These rates, respectively, describe the speciation of silicon in the silicate oligomers and the dilution of the silicate species. For $100 \mathrm{~g}$ of commercial solution, the total amount of the alkaline activation solution corresponding to the $\mathrm{R}_{1}$ and $\mathrm{R}_{2}$ ratios is:

$$
\begin{aligned}
\mathrm{Q}_{1} & =\frac{\mathrm{SiO}_{2} \text { rate in the commercial solution }}{\mathrm{SiO}_{2} \text { rate in the activation alcaline solution }} \\
& \star 100
\end{aligned}
$$

The amounts of $\mathrm{Na}_{2} \mathrm{O}, \mathrm{NaOH}$ and $\mathrm{H}_{2} \mathrm{O}$ are then defined by:

$$
\begin{aligned}
& \mathrm{Q}\left(\mathrm{Na}_{2} \mathrm{O}\right)=\left(\mathrm{Q}_{1} \star \% \mathrm{Na}_{2} \mathrm{O}\right) / 100-\% \mathrm{Na}_{2} \mathrm{O}(\mathrm{Sc}) \\
& \mathrm{Q}(\mathrm{NaOH})=2 \text { * }(\text { Masse Molaire }(\mathrm{NaOH})) /(\text { Masse } \\
&\text { Molaire } \left.\left(\mathrm{Na}_{2} \mathrm{O}\right)\right){ }^{\star} \mathrm{Q}\left(\mathrm{Na}_{2} \mathrm{O}\right)
\end{aligned}
$$
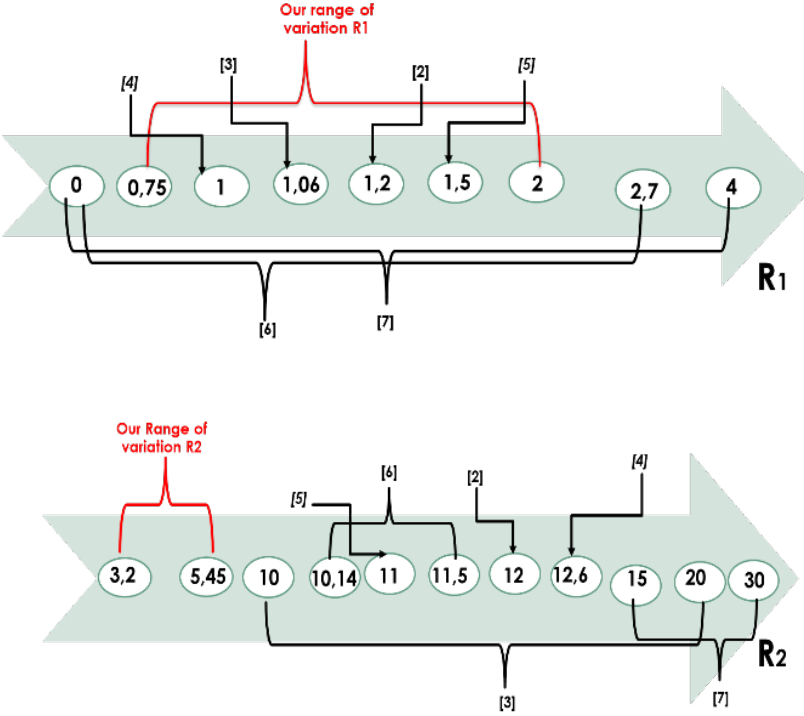

Figure 2: Position of our study in relation to the literature.

$$
\mathrm{Q}\left(\mathrm{H}_{2} \mathrm{O}\right)=\left(\mathrm{Q}_{1} \star \% \mathrm{H}_{2} \mathrm{O}\right) / 100-\% \mathrm{H}_{2} \mathrm{O}(\mathrm{Sc})
$$

This makes it possible to associate them in a one-toone way with the ratios $R_{1}$ and $R_{2}$. (Cf. Table 1 )

This approach, from which the correspondence between the weightings of the components and the structural parameters is established, makes it possible to go back to the importance of the impact of the percentage in sodium oxide on these parameters and to integrate, for a given value of this percentage, a set of cases for which the sum $\mathrm{R}_{1}+\mathrm{R}_{2}$ remains constant.

\subsubsection{Elaboration}

The sodium hydroxide pellets $(\mathrm{NaOH})$ are dissolved in distilled water under magnetic stirring of $6 \mathrm{tr} / \mathrm{s}$ for one hour before adding the commercial solution. The mixture thus obtained is solicited by the same agitation for two hours in 
order to ensure the chemical equilibrium between the silicated species. For this study, fifty samples are prepared. They correspond to the cases where $(11<\mathrm{P}<20.9)$ corresponding to $\left(0.75<R_{1}<2 ; 3.2<R_{2}<5.45\right)$. Compared to previous work and as shown in the figure (Figure 2), this is a deliberate choice justified by a desire to enrich the reflection around these formulations through the analysis of cases not yet addressed (formulations corresponding to low values of $R_{2}$ ).

\subsection{Case of metakaolin-based geopolymers}

\subsubsection{Formulation}

The solutions are obtained from the activation of a quantity $S$ of metakaolin by a quantity $L$ of an alkaline activation solution, under the conditions specified in the paragraph 1.2.1. This mixture is, therefore, characterized by the following structural parameters:

- The value $P$ of the percentage of sodium oxide (from which we can fix the ratios $R_{1}$ and $R_{2}$ ).

- The value of the ratio $R_{3}$.

In the following table (Table 2), we present $\mathrm{P}$ and $\mathrm{R}_{3}$ values selected for this study:

Table 2: values of $P$ and $R 3$

\begin{tabular}{cc}
\hline $\mathbf{P}$ & $\mathbf{R}_{3}=\mathrm{L} / \mathbf{S}$ \\
\hline $11.83 \%$ & 0.8 \\
$14.81 \%$ & 0.87 \\
$16.26 \%$ & 0.94 \\
$16.86 \%$ & 1.01 \\
\hline
\end{tabular}

\subsubsection{Elaboration}

After carrying out the necessary weightings of the solution and the powder, the two components are mixed using a kneader for $2 \mathrm{~min}$. This leads to the formation of a homogeneous paste that evolves chemically and rheologically over time $[3,6]$.

\section{Test Stand and Measurement Protocol}

\subsection{Description of the rheometer}

To describe the effects due to the formulation of the alkaline activation solution or the concentration of the aluminosilicate powder on the rheological behavior of the solutions obtained; we use, as a test bench, the "Haake Rheostress ${ }^{1 \text { " }}$ rotary rheometer from "Thermo scientific ${ }^{2 \text { " }}$ controlled by Rhéowin software and operating at imposed speed or stress. The tests are performed in plan-plane configuration for which the lower plane is fixed and the upper plane is rotated about their common axis; these planes being waterproof disks in Titanium 60mm diameter (PP60 cell), hardness coefficient equal to 6 (Figure 3) and gap equal to $1 \mathrm{~mm}$. This geometry adapts perfectly to the pasty nature of the material in question and requires small volumes for the samples $(2.9 \mathrm{ml})$.



Figure 3: Plan plan cellule

\subsection{Measurement protocol}

For this work, we will focus on the case where a triangular rotation signal, characterized by its rise time $\mathrm{T}_{0}$ and its maximum speed, is imposed on the upper disk (Figure 4). The ascending ramp is applied first to the material, which

1 Cadilhac est une entreprise de la distribution de produits chimiques et de produits d'entretien au Maroc. https://www.cadilhac.ma 2 Thermo scientific est une multinationale américaine fournissant du matériel de recherche et d'analyse aux laboratoires. https://www.thermofisher.com 




Figure 4: Imposed signal

is initially at rest, and is immediately followed by a descending ramp.

In a general way and for the rotary rheometers, it is established that the comparison of the answers to the two ramps is likely to inform us as to the flow regime. With the help of an asymptotic treatment of the conservation equations, we are indeed able to verify for the case where the flow is supposed to be quasi stationary (phenomena of inertia neglected before the viscous phenomena), that these responses are superimposed and that the shear rate $\gamma$. and the shear stress $\tau$ are respectively defined by $\tau=\mathrm{A} \Gamma$ and $\gamma$. $=\mathrm{B} \Omega$ where $\mathrm{A}$ and $\mathrm{B}$ are constants characterizing the geometry of the measuring cell (for the cell PP60, A $=0.02357(\mathrm{~Pa}$ ( $\mu \mathrm{Nm}$ ) and $\mathrm{B}=188.50$ ) and $\Gamma$ the hydrodynamic torque in the gap [13-15]. Experimentally, the preliminary tests that we conducted to identify the conditions for which the inertial effects can be minimized have allowed to observe, as shown in (Figure 5), that the responses to the two ramps are almost identical for $\mathrm{T}_{0}$ greater than or equal to $300 \mathrm{~s}$ and for values of $\Omega$ max close to $30 \mathrm{rpm}$.

\section{Rheological Behavior: Analysis and Results}

\subsection{Case of alkaline activation solutions}

Qualitatively, the results obtained in this case are in agreement with previous work $[2,6,7]$. The behavior of these solutions remains Newtonian when one of the two ratios $R_{1}$ or $R_{2}$ is varied. As far as we are concerned, we present directly in Figure 6 the effects due to the variations of the percentage $P$ on the evolution of the shear stresses. For values of $\mathrm{P}$ varying between 11.83 and $20.26 \%$, we show that

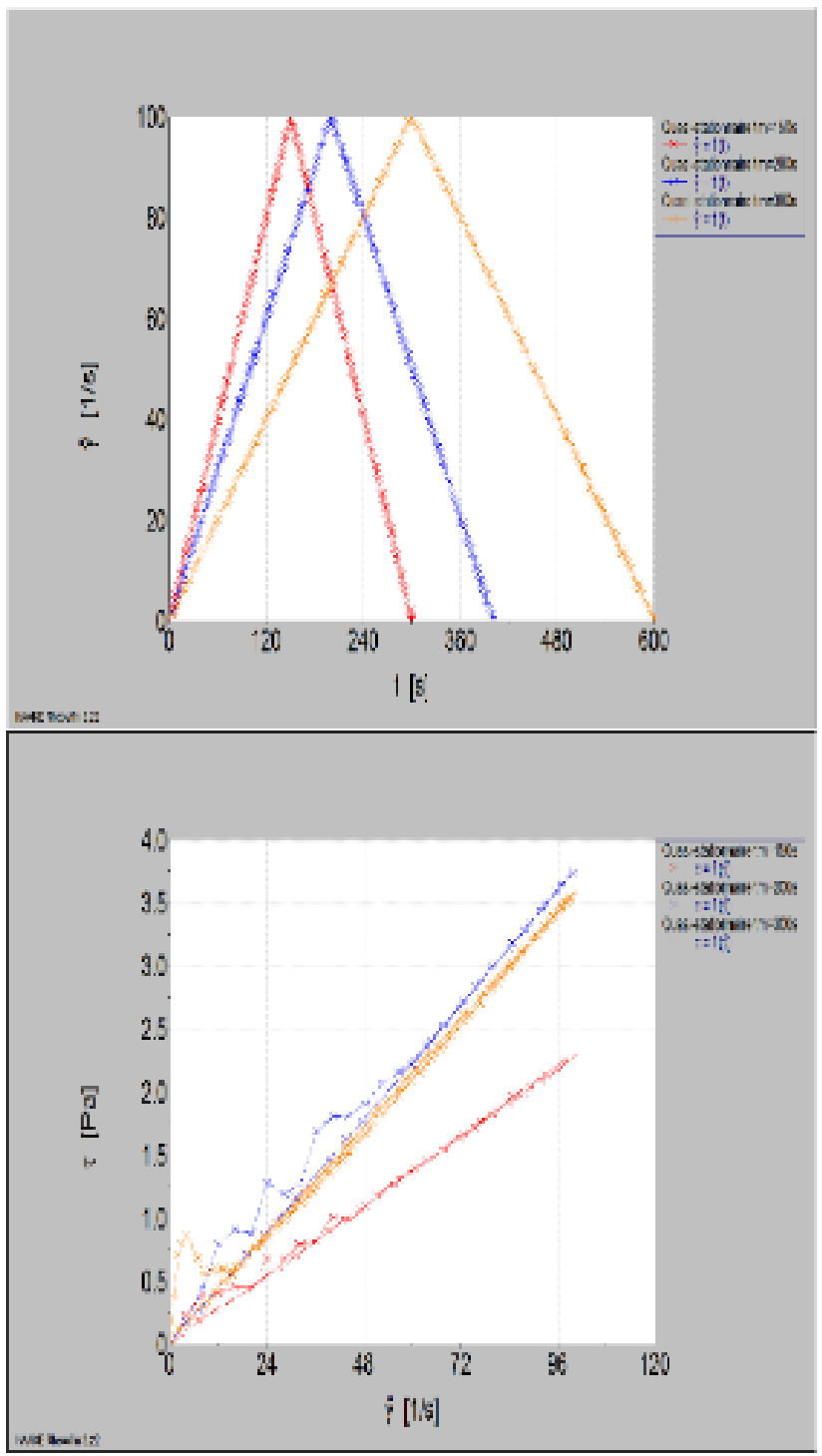

Figure 5: Determination of the rise time

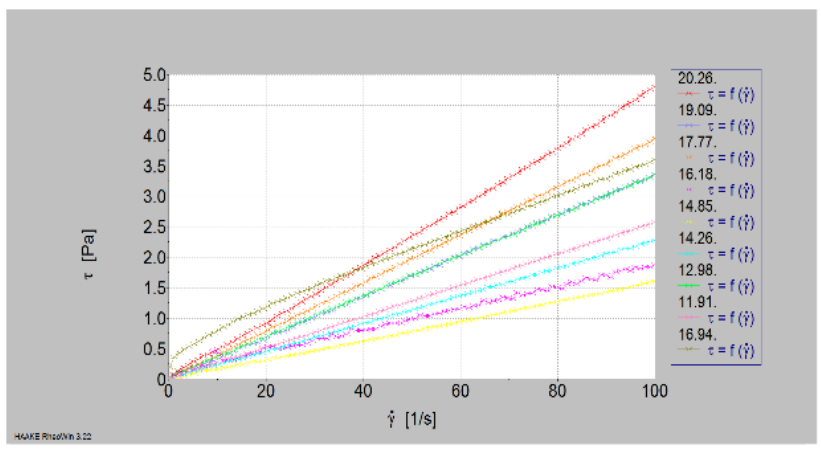

Figure 6: Rheological behavior of alkaline activation solution.

the viscosity of the alkaline activation solutions evolves as a polynomial of degree 5 and remains between 33 and $50 \mathrm{mPa} \cdot \mathrm{s}$. It should be noted that the inverse exploitation 




(a) Effect of P on the rheological behavior

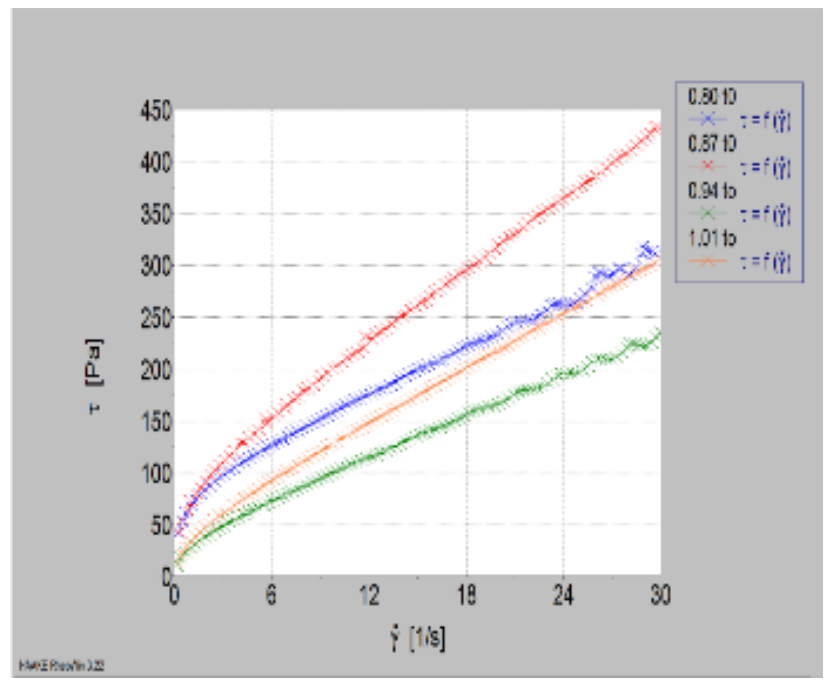

(b) Effect of L/S on the rheological behavior

Figure 7: Rheograms of the different formulations considered

of the plot of this variation would be, in our opinion, a considerable contribution to the prediction of the $P$ values and subsequently of the $R_{1}$ and $R_{2}$ ratios for a given solution [9].

\subsection{Case of geopolymers based on fresh metakaolin}

Numerous experimental studies $[2,3,6,7]$ have shown that the rheological behavior of fresh geopolymer matrices can be described by the Herschel-Bulkley model, defined by the relation:

$$
\tau=\tau_{0}+k(\dot{\gamma})^{n}
$$
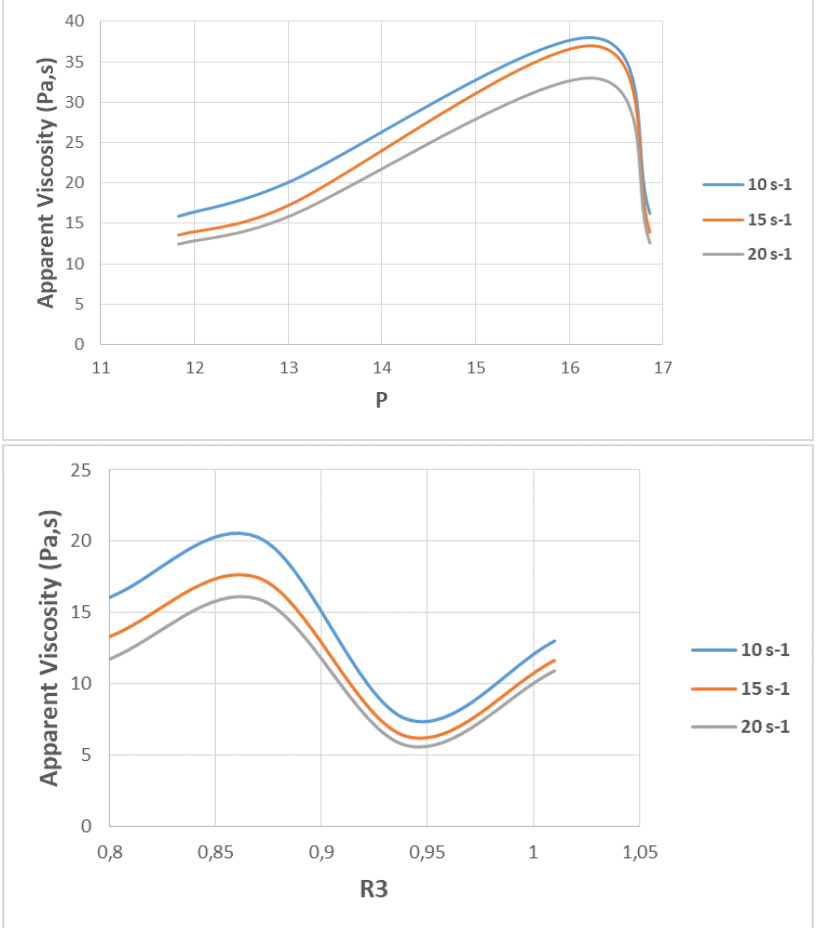

Figure 8: Evolutions of the apparent viscosity for a different shear rate and as function of $P$ and $R 3$.

With:

$\tau$ : The shear stress in $(\mathrm{Pa})$

$\tau_{0}$ : The yield stress $(\mathrm{Pa})$

$\mathrm{k}$ : The consistency $(\mathrm{Pa} \cdot \mathrm{s})$

$\dot{\gamma}$ : The shear rate (s-1)

$\mathrm{n}$ : The fluidity index

The tests that we carry out here make it possible to reinforce this result, they allow moreover the highlighting of the impact of the structural characteristics $\left(P, R_{3}\right)$ [18] of the geopolymer matrix on the rheological parameters $\left(\tau_{0}\right.$, $k$ and $n$ ) from changes in apparent viscosity (Figure 7a). In this context, it should be noted that contrary to the observations noted by Bourlon, the increase in the shear rate is accompanied in our case by a decrease in the apparent viscosity. This is due to the fact that the values we use for $R_{2}$ are very low compared to those considered by Bourlon. A [7] (Figure 7b).

For more details, we show in the figures (Figure 7), the different impacts due to the variations of these different quantities. This reinforces for the users of this type of matrix additional control means allowing more correlation between the structure of the solutions and their macroscopic behavior in the fresh state.

An important point at this level is concerns the observations related to the evolution of the apparent viscosity as a function of the percentage of sodium oxide or also ac- 


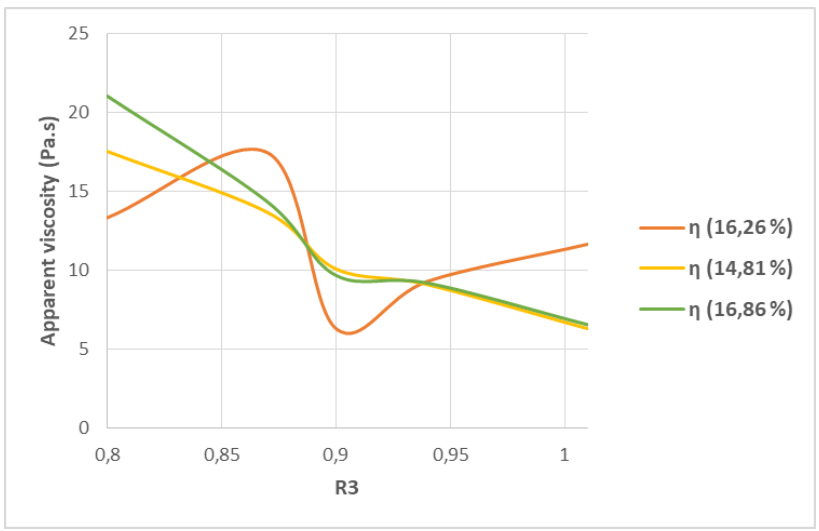

Figure 9: Effect of $\mathrm{L} / \mathrm{S}$ on the apparent viscosity

cording to the ratio $\mathrm{R}_{3}$. The appearance of the peak at the level of $P=16.26$ and $R_{3}=0.94$ is still valid for different shear rates (Figure 8 ), these modeling parameters represent a critical point characterizing the material.

It should be noted that during the execution of the rheological tests, we were able to determine critical points characterizing the rheology of the geopolymers. According to the figure (Figure 9), the existence of two points of intersection between the apparent viscosity curves of the different percentages of sodium oxide as a function of $R_{3}$ is very significant. This reinforces the fact that this percentage only slightly affects the apparent viscosity for these two values of $R_{3}$. These two cases correspond to values of $\mathrm{R}_{3}<1$.

We are able to further describe how, for the same ratio $\mathrm{R}_{3}$, the setting kinetics characterizing the start of the transition to the cured state, is directly affected by the formulation of the alkaline activation solution. This reinforces the conclusions of Wang et al. [4] on the possibilities of predicting the mechanical behavior of the finished product from the rheological properties of the alkaline activation solution $[2,3,6]$. This also confirms the remarks of $\mathrm{Xu}$ et al. [6] who were interested in studying the effect of the formulation of this solution on the mechanical strength of the geopolymer matrices in the cured state.

\subsection{Impact of the structural modeling on the rheological parameters $\left(\tau_{0}, k\right.$ and $\left.n\right)$}

The purpose of this paragraph is to highlight the effects due to the variations of the structural parameters $\left(\mathrm{P}, \mathrm{R}_{3}\right)$ on the rheology of the geopolymer matrix in the fresh state. And this in order to link later this rheological behavior to the kinetics of taking [19]. This constitutes to our senses

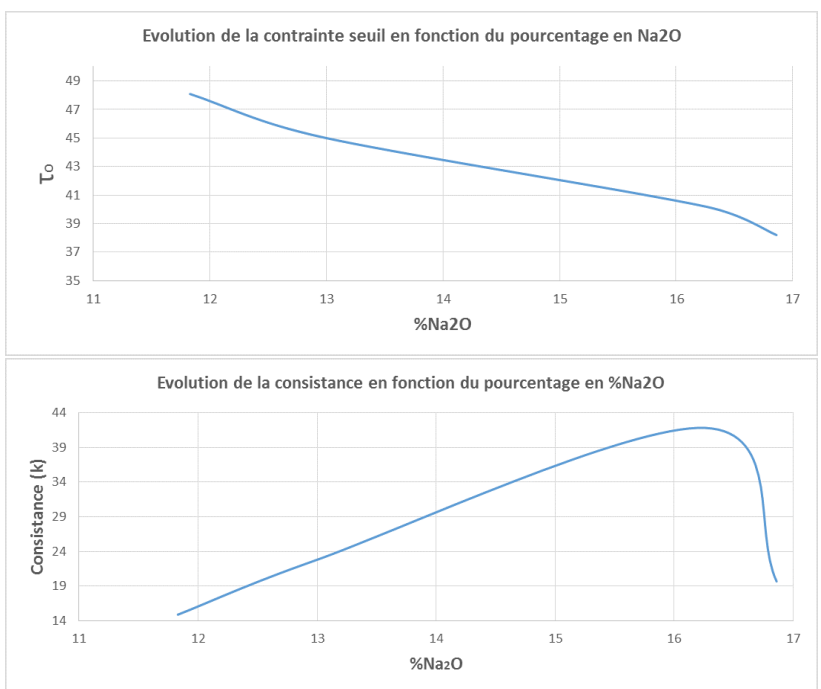

Evolution de l'indice de fluidité en fonction du pourcentage en $\mathrm{Na2O}$

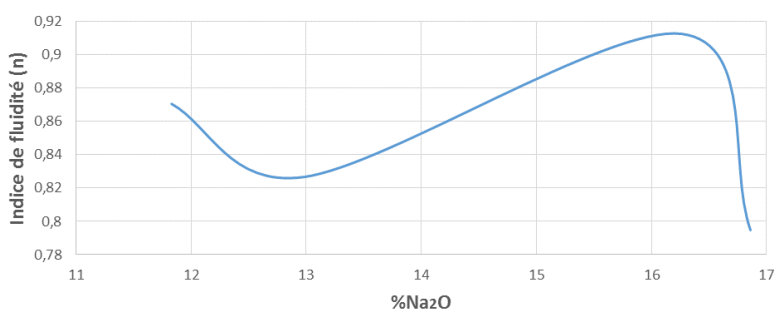

Figure 10: Effect of $P$ on the rheological parameters

a definite advance for the control of the formulations of these matrices in the fresh state, as we will verify later.

In this context, it is necessary to set the value of the shear rate to be able to record, at the level of the apparent viscosity, the effects due to the variation of the structural parameters on the values of the stress, the consistency and the index fluidity of these solutions.

\subsubsection{Effect of percentage of sodium oxide}

For a fixed shear rate $\dot{\gamma}=15 \mathrm{~s}^{-1}$, the evolution of the viscosity as a function of $\mathrm{P}$ makes it possible to record a substantial decrease in the yield stress, which is in accordance with the impact of the solution of alkaline activation on the behavior of geopolymer matrices. In addition, it allows to observe the existence of a peak around the value $\mathrm{P}=16.26$ $\left(R_{1}=1.2, R_{2}=4.017\right)$ for the evolutions of $k$ and $n$. This case for which $R_{3}=0.87$ seems to correspond to a particular situation that we consider later (Figure 10). 

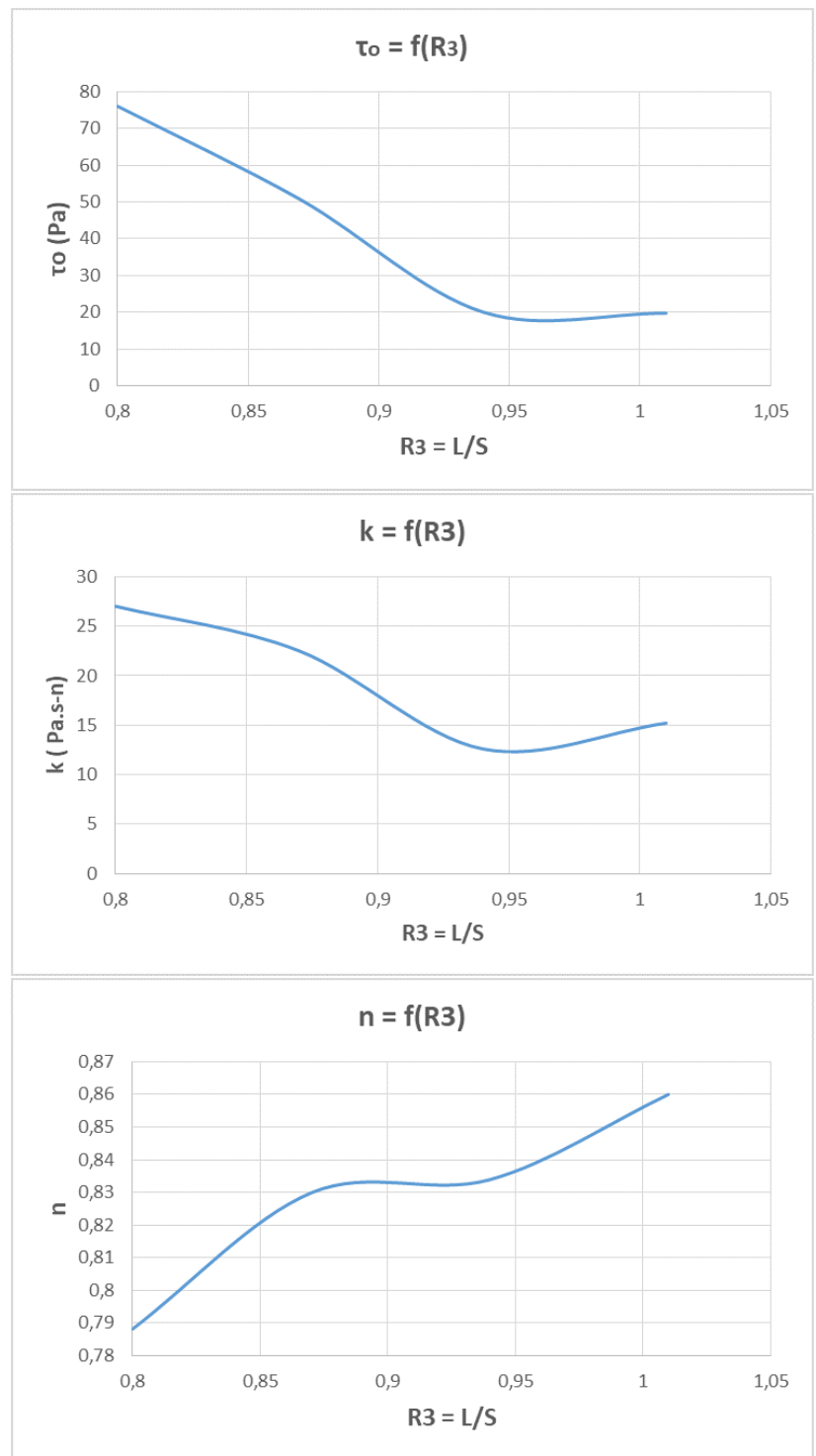

Figure 11: Effect of $\mathrm{L} / \mathrm{S}$ on the rheological parameters

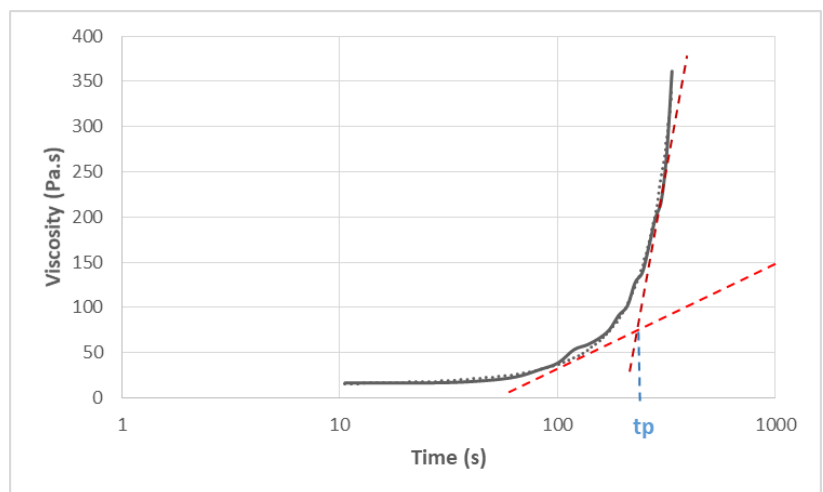

Figure 12: The tangent method for determining the setting time

\subsubsection{Effect of the ratio $R_{3}$}

For $\mathrm{P}=16.26$ and $\dot{\gamma}=15 \mathrm{~s}^{-1}$, the figure (Figure 11) shows that the evolution of quantities $\left(\tau_{0}, \mathrm{k}\right.$ and $\left.\mathrm{n}\right)$ is directly affected by the value of $R_{3}$. In particular, the corresponding curves show a concavity change around the value $R_{3}=0.94$.

\section{Approach for optimizing the formulation of geopolymers in the fresh state}

The quality of the geopolymers in the fresh state is practically linked to its kinetics of setting which directly delimits the start of the condensation process [17] and which also makes it possible to fix the modalities of their exploitation or their transport. In view of the results presented, our approach for the optimization of the formulation of fresh geopolymer matrices is built on the basis of changes in setting time as a function of the parameters that are likely to affect them. Note that this is essentially the percentage of sodium oxide $(\mathrm{P})$, the $\mathrm{L} / \mathrm{S}$ ratio $\left(\mathrm{R}_{3}\right)$ and the temperature. We therefore place ourselves in the case where $R_{3}<1$ and we seek to determine the optimized version of our formulation which allows to have the longest setting time. It is noted that for the measurement of the setting time, we consider the tangents method (Figure 12) when the evolution of the viscosity over time is broken down into two parts, one having the first stages where the viscosity remains almost constant and the second where the viscosity follows an inflection and grows rapidly. This setting phenomenon, which characterizes the percolation path, is due to the bond between siliceous and aluminous oligomers.

\subsection{Effect of the temperature on setting kinetics}

For the effect of temperature, we consider the case where $\mathrm{T}=40^{\circ} \mathrm{C}$ (closest to ambient temperature), simply because when the temperature is raised, the set time decreases. As has been confirmed by the previous work $[2,3,6]$ and as has been verified through the evolution shown in Figure 13. Indeed, for a given formulation; $\mathrm{P}=16.86$ and $\mathrm{R}_{3}=0.87$, the evolution of setting time as a function of temperature is predictable. Note that the temperature which corresponds to the longest setting time allowing a favorable condition for its flow is $40^{\circ} \mathrm{C}$ (Figure 13). 


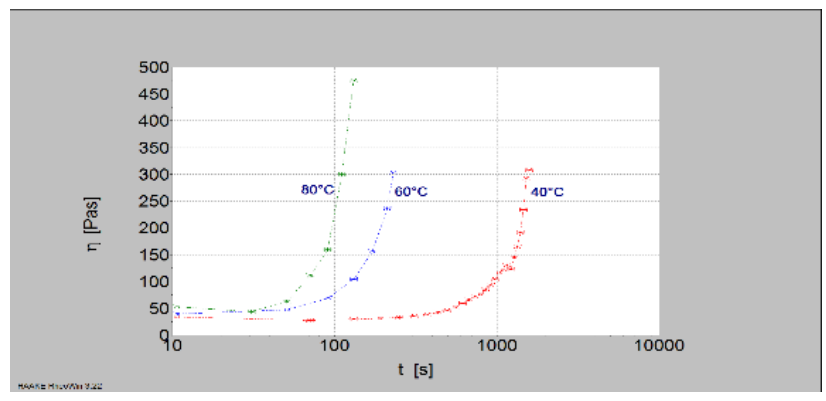

Figure 13: Effect of the temperature on the setting time

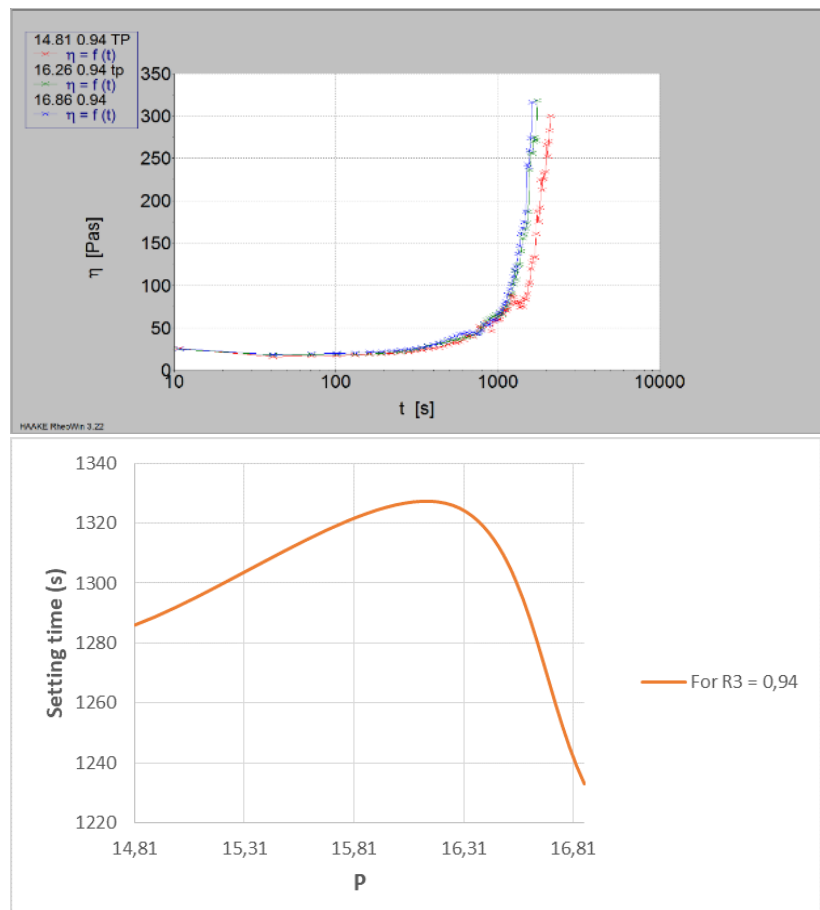

Figure 14: Evolution of the setting time for a different $\mathrm{P}$ and for $\mathrm{L} / \mathrm{S}=$ 0.94

\subsection{Effect of the sodium oxide percentage on the setting time of geopolymers}

For the ratio $\mathrm{R} 3=0.94$ fixed, the appearance of a peak when $\mathrm{P}=16.26$ reinforces the results above (Figure 11). This percentage has a peculiarity in the rheological properties, it is a maximum of apparent viscosity and setting time (Figure 14).

\subsection{Effect of the ratio $R_{3}=L / S$ on the setting time of the geopolymers}

The study of this effect seems to be useful and necessary in order to define the quantity of the alkaline solution suitable for activating an amount of the aluminosilicate pow-



Figure 15: Evolution of the setting time for a different $\mathrm{L} / \mathrm{S}$ and for $\mathrm{P}=$ $16.26 \%$

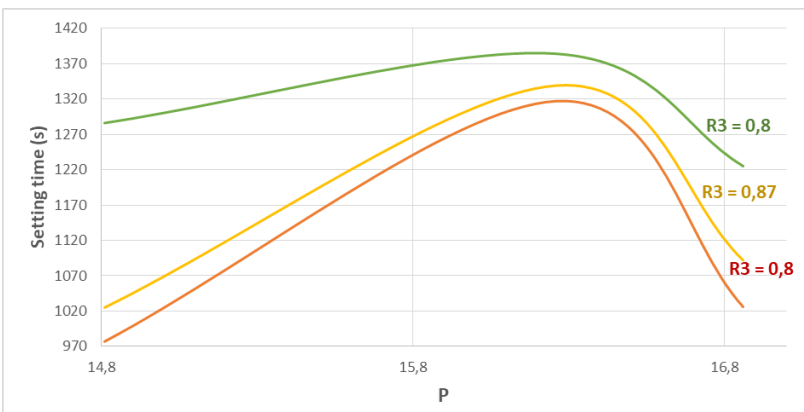

Figure 16: Effect of the structural parameters on the setting time

der on the one hand and to specify the desired setting time. For $\mathrm{P}=16.26$, a point of inflection and concavity change that occurs at the ratio 0.94 (Figure 15).

From the above curves, the slowest setting time is that containing $\mathrm{P}=16.26 \%$ and $\mathrm{L} / \mathrm{S}=0.94$ (Figure 16). This is of particular importance in determining the flow time.

Then, it is possible to describe how, for the same ratio R3, the setting kinetics characterizing the start of the transition to the cured state, is directly affected by the for- 
mulation of the alkaline activation solution. These observations reinforce the conclusions of Wang et al. [10] on the possibilities of predicting the mechanical behavior of the finished product from the rheological properties of the alkaline activation solution $[2,3,6]$. These also confirm the remarks of Xu et al. [6] who were obtained in studying the effect of the formulation of this solution on the mechanical strength of the geopolymer in the cured state.

Table 3: the values of the correspondence between the apparent viscosity and the setting time

\begin{tabular}{cccc}
\hline$\% \mathrm{Na}_{2} \mathbf{O}$ & L/S & $\boldsymbol{Y}_{\text {app }}(\mathrm{Pa} \cdot \mathbf{s})$ & Setting time (s) \\
\hline 14.81 & 0.8 & 17.53 & 498.1 \\
& 0.87 & 13.67 & 539.9 \\
& 0.94 & 10.09 & 1286 \\
\hline 16.26 & 0.8 & 13.32 & 812.5 \\
& 0.87 & 17.45 & 1020 \\
& 0.94 & 6.325 & 1340 \\
\hline 16.86 & 0.8 & 21.03 & 531.78 \\
& 0.87 & 14.34 & 612.2 \\
& 0.94 & 9.685 & 1233 \\
\hline
\end{tabular}

We present in Table 3 the corresponding values of the apparent viscosity as well as the setting time. In particular, it is observed that for the optimum formulation proposed in the preceding paragraph, the apparent viscosity is the lowest when the setting time is longer.

\section{Conclusion}

For the study of the rheological behavior of geopolymer materials based on metakaolin, we, initially, justified the choice of the structural parameters that we selected for the identification of the formulations considered. These parameters are: $\mathrm{P}=\% \mathrm{Na}_{2} \mathrm{O}$ and $\mathrm{R}_{3}=\mathrm{L} / \mathrm{S}$, deduced from a simple conversion relation. To highlight the impact of the percentage of sodium oxide on $\mathrm{R}_{1}$. $=\mathrm{SiO}_{2} / \mathrm{Na}_{2} \mathrm{O}$ and $\mathrm{R}_{2}$ $=\mathrm{Na}_{2} \mathrm{O} / \mathrm{H}_{2} \mathrm{O}$ (usually considered) and to integrate, for a given value of $P$, a set of cases for which the sum $R_{1}+R_{2}$ remains constant. A particular interest was devoted to the analysis of the impact of these parameters on the rheological behavior of these solutions. For the different cases considered, we have shown that:

- The rheological behavior is described by Herschel Bulkley's three-parameter viscoplastic model (yield stress $\tau_{0}$, consistency $\mathrm{k}$ and melt flow index $\mathrm{n}$ ).
- The yield stress decreases when P increases, while the consistency and the melt index have a maximum around $\mathrm{P}=16.26 \%$.

- The changes in the rheological parameters $\left(\tau_{0}, \mathrm{k}\right.$ and $n$ ) as a function of $R_{3}$ show a change of curvature around $\mathrm{R}_{3}=0.94$

For more control of the evolution of this rheological behavior we followed, with the help of the temporal variations of the viscosity, the beginning of the transition towards the hardened state. It was then observed that:

- Setting time $\left(\mathrm{t}_{p}\right)$ decreases with temperature.

- The variation of $\left(t_{p}\right)$ as a percentage of sodium oxide has a maximum around $\mathrm{P}=16.26 \%$.

- The variation of $\left(t_{p}\right)$ as a function of the $\mathrm{L} / \mathrm{S}$ ratio has a point of inflection around $R_{3}=0.94$.

Given these different results, we are able to propose an optimized formulation of the analyzed solutions. This corresponds to the case where the setting time is the longest $\left(t_{p}=1340 \mathrm{~s}\right)$ or else that for which $\mathrm{P}=16.26 \%$ and $\mathrm{R}_{3}=0.94$. For this formulation, we obtained the lowest values of the apparent viscosity. This is consistent with the results observed in terms of setting time.

This consolidates the processes of control of the rheology of geopolymers and the prediction of the behavior in the hardened state.

Conflict of Interests: The authors declare that there is no conflict of interest.

Acknowledgement: Appel à projetsautour des phosphates APPHOS - sponsored by OCP (OCP Foundation, R\&D OCP, Mohammed VI Polytechnic University, National Center of Scientific and technical Research CNRST, Ministry of Higher Education, Scientific Research and Professional Training of Morocco MESRSFC) under the project entitled * Matériaux de construction à base de matrices géopolymères et d'agrégats sous-produits de l'industrie des phosphates ${ }^{\star}$, project ID * MAT-MOS-01/2017*

\section{References}

[1] Davidovits J., Geopolymer-Chemistry and Applications, 4th ed., Institut Géopolymère, 2015

[2] Duxon P., Mallicoat S.W., Lukey G.C., Kriven W.M., van Deventer J.S.J., The effect of alkali and $\mathrm{Si} / \mathrm{Al}$ ratio on the development of mechanical properties of metakaolin-based geopolymers, J. Colloids and Surfaces A: Physicochemical and Engineering Aspects, DOI: 10.1016/j.colsurfa.2006.05.044 
[3] Rowles M., O'Connor B., Chemical optimization of the compressive strength of aluminosilicate geopolymers synthesised by sodium silicate activation of metakaolinite, J. Mater. Chem. 13, 1161-1165.

[4] Wang J. W., Cheng T.W., Production geopolymer materials, Proceedings of the 7th International Symposium on East Asian Resources Recycling Technology (10-14 November 2003, Tainan, Taiwan), Tainan, 2003, 263-266

[5] Palomo A., Banfill R.F.G., Fernandez-Jimenez A., Swift D.S., Properties of alkali activated fly ashes determined from rheological measurements, J. Advances in Cement Research, DOI:10.1680/adcr.2005.17.4.143

[6] Steins P., Influence des paramètres de formulation sur la texturation et la structuration des géopolymères, $\mathrm{PhD}$ thesis, École doctorale Sciences et Ingénierie des Matériaux, Mécanique, Energétique et Aéronautique, Limoges, France, 2014

[7] Bourlon A., Physico-chimie et rhéologie de géopolymères frais pour la cimentation des puits pétroliers, $\mathrm{PhD}$ thesis, University Pierre et Marie Curie, Paris, France, 2010

[8] Favier A., Habert G., Baptiste d'Espinose de Lacaillerie J., Roussel N., Mechanical properties and compositional heterogeneities of fresh geopolymer pastes, J. cement and concrete research, DOI: 10.13140/2.1.4794.1444

[9] Aboulayt A., Riahi M., Ouazzani Touhami M. , Hannache H., Gomina M., Moussa R., Properties of métakaolin based geopolymer incorporating calcium carbonate, J. Advanced Powder Technology, DOI: 10.1016/j.apt.2017.06.022

[10] Lahlou N., Ouazzani Touhami M., Moussa R., Hattaf R., Effect of the Formulation of Sodium Activation Solutions on the Setting Time of Metakaolin Based Geopolymers, J. Materials Science and EngineeringB, DOI: 10.17265/2161-6221/2019.1-2.002
[11] Favier A., Mécanisme de prise et rhéologie de liants géopolymères modèles, $\mathrm{PhD}$ thesis, Paris-Est University, Paris, France, 2013

[12] Vail J. G., Willis J.H., soluble silicates - their properties and uses, 1st ed., New York, Reinhold, 1952

[13] Ouazzani Touhami M., Modélisation mathématique d'écoulements unidimensionnels de fluides viscoélastiques, PhD thesis, University Hassan II, Casablanca, Morocco, 1991

[14] Ovarlez G., Mahaut F., Deboeuf S., Lenoir N., Hormozi S., Château X., Flows of suspensions of particles in yield stress fluids, J. Rheology, DOI: $10.1122 / 1.4934363$

[15] Coussot P., Ovarlez G., Introduction- yield stress or 100 years of rheology, J. Rheologica Acta, DOI: 10.1007/s00397-017-1003-6

[16] Aboulayt A., Elaboration et caractérisation de matériaux géopolymeres à base de Kaolin, PhD thesis, University Hassan II, Casablanca, Maroc, 2014

[17] Poulesquen A., Rheological behavior of alkali-activated métakaolin during geopolymerization, In: Bart F., Cau-dit-Coumes C., Frizon F., Lorente S., (Eds.), Cement-Based Materials for Nuclear Waste Storage, 1st ed., Libgen Librarian, Springer-Verlag New York, 2013

[18] Krieger I. M., Dougherty T. J., A Mechanism for Non-Newtonian Flow in Suspensions of Rigid Spheres, J. Rheology, DOI: $10.1122 / 1.548848$

[19] Vyšvařil M., Vejmelková E., Rovnanikova P., Rheological and mechanical properties of alkaliactivated brick powder based pastes: effect of amount of alkali activator, J. Materials Science and Engineering, DOI: 10.1088/1757-899X/379/1/012011 